\title{
Fenologia reprodutiva e sistema de polinização de Ziziphus joazeiro Mart. (Rhamnaceae): atuação de Apis mellifera e de visitantes florais autóctones como polinizadores ${ }^{1}$
}

\author{
Tarcila de Lima Nadia ${ }^{2,4}$, Isabel Cristina Machado e Ariadna Valentina Lopes $^{3}$
}

Recebido em 20/10/2006. Aceito em 2/03/2007

\begin{abstract}
RESUMO - (Fenologia reprodutiva e sistema de polinização de Ziziphus joazeiro Mart. (Rhamnaceae): atuação de Apis mellifera e de visitantes florais autóctones como polinizadores). Ziziphus joazeiro é uma espécie endêmica da Caatinga, com grande utilidade econômica, cuja biologia reprodutiva é pouco conhecida. Este estudo aborda a fenologia reprodutiva, a biologia floral e o sistema de polinização de Ziziphus joazeiro no Cariri Paraibano, Nordeste do Brasil. Os períodos de floração e de frutificação ocorreram no fim da estação seca e início da chuvosa, com picos nos meses de dezembro (floração) e fevereiro (frutificação). As flores são do tipo disco, esverdeadas, duram cerca de 12 horas e possuem disco nectarífero largo e amarelo, o qual circunda o gineceu. Ocorre protandria associada a outro tipo de dicogamia, a heterodicogamia. Ziziphus joazeiro produz néctar em pequena quantidade ( $1 \mu \mathrm{l})$, com alta concentração de açúcares (75\%). Os visitantes florais observados foram vespas, abelhas e moscas. Apis mellifera apresentou maior freqüência de visitas (77,5\%), seguida pelo grupo das vespas $(20,4 \%)$, ambos considerados polinizadores efetivos. As moscas e as outras espécies de abelhas apresentaram baixo percentual de visitas $(2,1 \%)$ e foram consideradas pilhadoras de néctar. A elevada taxa de desenvolvimento inicial de frutos por inflorescência pode sugerir alta eficiência dos polinizadores, uma vez que o mecanismo de dicogamia presente na espécie praticamente impede a ocorrência de autopolinização espontânea e de geitonogamia.
\end{abstract}

Palavras-chave: Ziziphus joazeiro, Apis, vespas, melitofilia, Caatinga

\begin{abstract}
Reproductive phenology and pollination system of Ziziphus joazeiro Mart. (Rhamnaceae): the role of Apis mellifera and autochthonous floral visitors as pollinators). Ziziphus joazeiro is an endemic species of the Caatinga with great economic importance, whose reproductive biology is poorly understood. This paper analyses the reproductive phenology, floral biology and pollination system of Ziziphus joazeiro at Cariri Paraibano, northeastern Brazil. Flowering and fruiting periods occurred at the end of the dry season and beginning of the rainy season, with peaks in December (flowering) and February (fruiting). The green, dish-shaped flowers last about 12 hours and have a large yellow nectary surrounding the gynoecium. Protandry occurs associated with heterodichogamy, another type of dichogamy. Ziziphus joazeiro produces little nectar $(1 \mu \mathrm{l})$ but with a high sugar concentration (75\%). Floral visitors were wasps, bees and flies. Apis mellifera had the highest frequency of visits (77.5\%), followed by wasps (20.4\%), both acting as effective pollinators. Flies and other bee species had a lower percentage of visits $(2.1 \%)$ and acted as nectar robbers. The high rate of initial fruit development per inflorescence suggests high pollinator efficiency, since the dichogamic mechanism prevents the occurrence of spontaneous selfpollination and geitonogamy.
\end{abstract}

Key words: Ziziphus joazeiro, Apis, wasps, melittophily, Caatinga

\section{Introdução}

A família Rhamnaceae é composta por cerca de 58 gêneros e 900 espécies com distribuição cosmopolita, sendo representada por 23 gêneros e 170 espécies na América Tropical (Heald 2004). Ziziphus possui cerca de 30 espécies (Heald 2004), seis das quais ocorrem na região Nordeste do Brasil (CNIP 2005). Ziziphus joazeiro, popularmente conhecida como juazeiro, é endêmica da Caatinga (Giulietti et al. 2002), apresentando grande importância econômica e ecológica, sendo utilizada localmente para produção de lenha e carvão, arborização de ruas e jardins, além de possuir frutos comestíveis (Lorenzi 1998), os quais são explorados de forma extrativista. Por ser uma das poucas espécies de Caatinga que se conservam sempre verdes (Braga 1976), as folhas de Z. joazeiro podem ser utilizadas na alimentação de caprinos e ovinos como

\footnotetext{
Parte da Dissertação de Mestrado da primeira Autora

2 Universidade Federal de Pernambuco, Programa de Pós-Graduação em Biologia Vegetal, 50372-970 Recife, PE, Brasil

Universidade Federal de Pernambuco, Departamento de Botânica, 50372-970 Recife, PE, Brasil

4 Autor para correspondência: tarcinadia@yahoo.com.br
} 
um recurso alternativo durante a época seca (Barros et al. 1991). Suas flores são importante fonte de recurso alimentar para abelhas indígenas sem ferrão da tribo Meliponini, as quais são utilizadas na meliponicultura, sendo atividade alternativa de renda para produtores de algumas áreas de Caatinga (Marinho et al. 2002).

Apesar da importância ecológica da espécie, pouco se conhece sobre sua biologia reprodutiva. Marinho et al. (2002) e Aguiar et al. (2003), estudando recursos florais utilizados por abelhas Meliponini e Centridini em Caatinga, citam $Z$. joazeiro entre as espécies listadas, sem fornecer detalhes acerca da ecologia da polinização dessas espécies. Pinheiro et al. (1991), por sua vez, registraram apenas uma abelha não nativa (Apis mellifera) como o polinizador efetivo de populações naturais de Z. joazeiro ocorrentes, contudo, em áreas referidas pelas autoras como bastante devastadas, no estado do Ceará, nordeste do Brasil.

É interessante, portanto, um estudo detalhado sobre a biologia floral e os polinizadores autóctones desta espécie. Tal conhecimento científico poderá auxiliar futuros trabalhos sobre conservação e manejo, tanto de $Z$. joazeiro como de seus polinizadores, bem como incremento na sua produção e uso racional dos seus recursos. Assim, os objetivos deste estudo foram analisar a fenologia reprodutiva e a biologia floral de Ziziphus joazeiro em uma área de Caatinga nativa, bem como verificar o papel de Apis mellifera e dos seus demais visitantes florais como polinizadores, discutindo a importância destes para o sucesso reprodutivo da espécie.

\section{Material e métodos}

Local de estudo - As observações de campo e coleta de dados foram feitas na Fazenda Dona Soledade, com cerca de 690 ha, localizada a $550 \mathrm{~m}$ de altitude no Planalto da Borborema, entre os municípios de Boa Vista e Cabaceiras $\left(7^{\circ} 20^{\prime} 30,7^{\prime}\right.$ 'S e $\left.36^{\circ} 18^{\prime} 5,8^{\prime \prime} \mathrm{W}\right)$, Estado da Paraíba, Nordeste do Brasil. A fazenda está localizada em região de Caatinga denominada como Região do Cariri, apresentando precipitação média anual de aproximadamente $330 \mathrm{~mm}$, com período chuvoso concentrado nos meses de fevereiro a maio (Governo da Paraíba 2005). A vegetação do Cariri é caracterizada como sendo Caatinga Arbustiva Densa ou Aberta, possuindo o registro mais baixo de chuvas das caatingas (Prado 2003). A área estudada apresenta como gêneros mais comuns Pilosocereus Byles \& Rowley (Cactaceae), Jatropha L. (Euphorbiaceae),
Mimosa L., Caesalpinia L. (Leguminosae), Schinopsis Engl., Myracrodruon Allem. (Anacardiaceae) e Aspidosperma Mart. \& Zucc. (Apocynaceae).

Fenologia reprodutiva e biologia floral - Foi feito acompanhamento da fenologia reprodutiva quinzenalmente em nove indivíduos de Ziziphus joazeiro, no período de agosto/2004 a julho/2005. O percentual de flores e frutos foi registrado segundo metodologia de Fournier (1974), obtendo-se a intensidade de floração e frutificação (Bencke \& Morellato 2002). Os padrões fenológicos de floração foram determinados a partir das classificações de Gentry (1974) e Newstrom et al. (1994).

O número de flores por inflorescência foi registrado, no campo, em 30 inflorescências ainda jovens de cinco indivíduos diferentes, contando-se o número de flores abertas e botões. A duração da antese foi monitorada em campo, marcando-se botões em préantese, sendo estes acompanhados até a senescência floral. A receptividade do estigma foi testada em campo utilizando-se solução 0,25\% de permanganato de potássio $\left(\mathrm{KMnO}_{4}\right)($ Robinsohn 1924). Os testes foram feitos tanto no início quanto seis horas após o início da antese, em dez flores de cinco indivíduos diferentes. Vinte flores, desses mesmos indivíduos, foram colocadas em recipiente de vidro com tampa para verificar emissão de odor. Para localização de áreas de concentração de emissão de odor, outras 10 flores, de cinco diferentes indivíduos, previamente ensacadas, foram submersas em vermelho neutro, lavadas posteriormente com água destilada e analisadas quanto às regiões coradas (Vogel 1990; Dafni 1992; Kearns \& Inouye 1993).

O volume e a concentração de açúcares no néctar foram medidos, em flores previamente ensacadas, com microsseringas ( $5 \mu \mathrm{l}$ e $25 \mu$ l Microliter $\left.{ }^{\circledR}\right)$ e refratômetros de bolso $\left(0-32 \%, 28-62 \%\right.$ e $58-90 \%$, Atago $\left.^{\circledR}\right)$, respectivamente. $\mathrm{O}$ néctar foi retirado de dez flores de diferentes indivíduos ao final da fase masculina e ao final da fase feminina, utilizando sempre as mesmas flores, e, em outras dez flores, ao final de antese (volume acumulado de néctar).

Flores de cinco indivíduos de Ziziphus joazeiro foram coletadas para análise da morfologia floral $(n=20)$ em laboratório, sob estereomicroscópio. O número de grãos de pólen por antera foi contado em cinco botões em pré-antese, de cinco indivíduos diferentes (utilizando-se todas as anteras do botão), através de contagem direta, uma vez que o número de grãos por antera é menor que 2.000 (Cruden 1977), 
com auxílio de lamínula quadriculada e microscópio óptico. Esses mesmos grãos de pólen foram corados com carmim acético $1,2 \%$ para verificar a viabilidade polínica (Radford et al. 1974).

Visitantes florais e disponibilidade polínica - O comportamento dos visitantes florais foi observado no campo em indivíduos focais e analisado com auxílio de fotografias. Estes foram classificados como polinizadores ou pilhadores, caso efetuassem ou não a polinização durante as visitas às flores. O local de deposição de pólen no corpo do animal visitante foi analisado de acordo com o seu comportamento de visita. A atuação da abelha Apis mellifera como polinizadora de Ziziphus joazeiro foi verificada ensacando-se botões em pré-antese $(n=46)$, os quais durante a antese foram expostos a no mínimo cinco visitas feitas exclusivamente por Apis mellifera durante a fase feminina da flor, sendo as flores posteriormente ensacadas. Após 24 horas, essas flores foram coletadas e fixadas em $\mathrm{FAA}_{70}$ para serem analisadas quanto ao número de grãos de pólen depositados no estigma e ao número de tubos polínicos, sob microscópio de fluorescência, seguindo técnica de Martin (1959). Alguns indivíduos de cada espécie visitante foram coletados e montados a seco para análise de suas estruturas e identificação, sendo depositados no Laboratório de Biologia Floral e Reprodutiva, da Universidade Federal de Pernambuco. O comprimento do corpo dos visitantes florais capturados foi medido com régua milimetrada da extensão da cabeça ao abdômen.

A frequiência de visitas foi calculada no início, pico e fim de floração de cada planta focal durante 13 horas consecutivas para cada período, contadas a partir das 5:00 h. Para o cálculo da frequiência de visitas, cada espécie visitante foi considerada individualmente, exceto para as moscas, que foram consideradas em grupo, devido à dificuldade de identificação no campo durante as visitas. Uma visita significou cada ato de procura pelo recurso floral por um dado visitante, ao pousar em uma das flores da inflorescência. A freqüência de visitas também foi calculada para flores que permaneciam na inflorescência durante o primeiro, o segundo e o terceiro dias após o início da antese. Duas flores de cada dia foram marcadas e acompanhadas quanto ao número de visitas recebidas, sendo essas observações feitas em um período de duas horas consecutivas em cada fase sexual. No total foram realizadas 82 horas de observação exclusivamente para freqüência.
A disponibilidade de pólen durante a antese foi verificada coletando-se duas flores, não ensacadas, a cada três horas, do início ao fim da antese, totalizando 10 flores/indivíduo $(\mathrm{n}=2$ indivíduos em pico de floração). Os grãos de pólen foram retirados de todas as anteras de cada flor, colocados em lâmina histológica, corados com carmim acético e fixados com glicerina. Posteriormente, foram contados diretamente sob estereomicroscópio.

Eficácia reprodutiva - Para verificar a eficácia reprodutiva através da atuação dos polinizadores (sensu Zapata \& Arroyo 1978) foi marcado um total de seis inflorescências/indivíduo, de cinco indivíduos diferentes ( $n=30$ inflorescências), cujas flores foram contadas e observadas quanto à formação de frutos sob condições naturais. Outras 15 inflorescências foram ensacadas, nos mesmos cinco indivíduos (três/indivíduo), para realizar polinizações cruzadas manuais em cerca de $25 \%$ das flores de cada inflorescência à medida que as mesmas se abriam. $\mathrm{O}$ índice de eficácia reprodutiva foi então calculado através da razão entre a percentagem de frutos formados em condições naturais (controle) e da percentagem de frutos formados através de polinização cruzada manual (sensu Zapata \& Arroyo 1978).

Análises estatísticas - Foi utilizado o teste de Lilliefors para verificar a normalidade dos dados. Para verificar diferenças estatísticas entre o volume de néctar produzido nas diferentes fases sexuais foi utilizado o teste de Mann-Whitney. Para comparações da freqüência de visitas em diferentes fases sexuais e entre os experimentos de eficácia reprodutiva foi utilizado o qui-quadrado. Por último, foi feita a correlação de Spearman entre o número de flores e de frutos por inflorescência, formados em condições naturais. As análises estatísticas foram feitas com auxílio do BioEstat 3.0 (Ayres et al. 2003).

\section{Resultados}

Fenologia reprodutiva e biologia floral - A floração de Ziziphus joazeiro teve início no mês de novembro, com pico no mês de dezembro, correspondente à estação seca (Fig. 1), prolongando-se com baixa intensidade no período chuvoso até meados de junho. Os frutos começaram seu desenvolvimento em meados de dezembro, ainda no fim da estação seca, atingindo a maior intensidade durante o período chuvoso, entre os meses de fevereiro a maio, quando se apresentavam maduros (Fig. 1). 


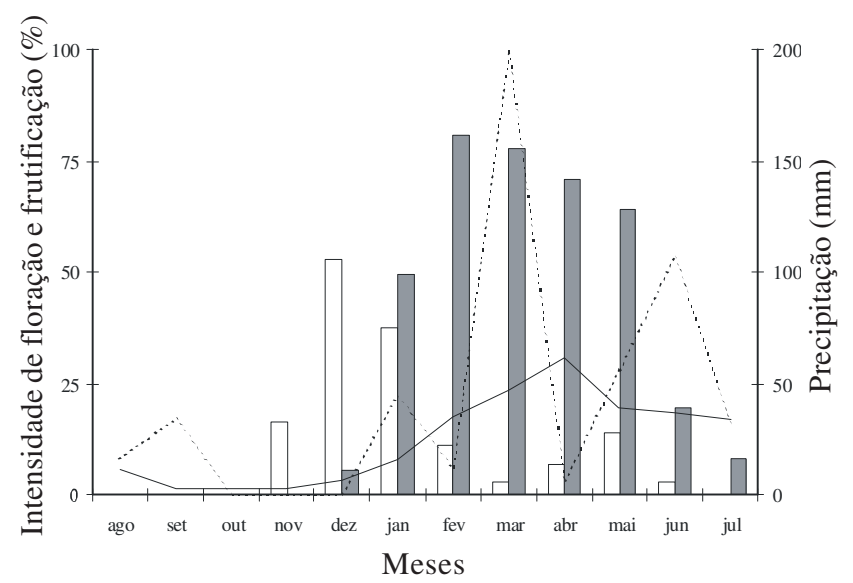

Figura 1. Intensidade de floração ( $\square$ ) e frutificação ( $\square$ ) de Ziziphus joazeiro Mart. (Rhamnaceae) durante o período de agosto/2004 a julho/2005, na fazenda Dona Soledade, municípios de Boa Vista e Cabaceiras, Paraíba. Precipitação pluviométrica mensal histórica (一) e entre o período de agosto/2004 a julho/2005 (---) do município de Boa Vista/PB.
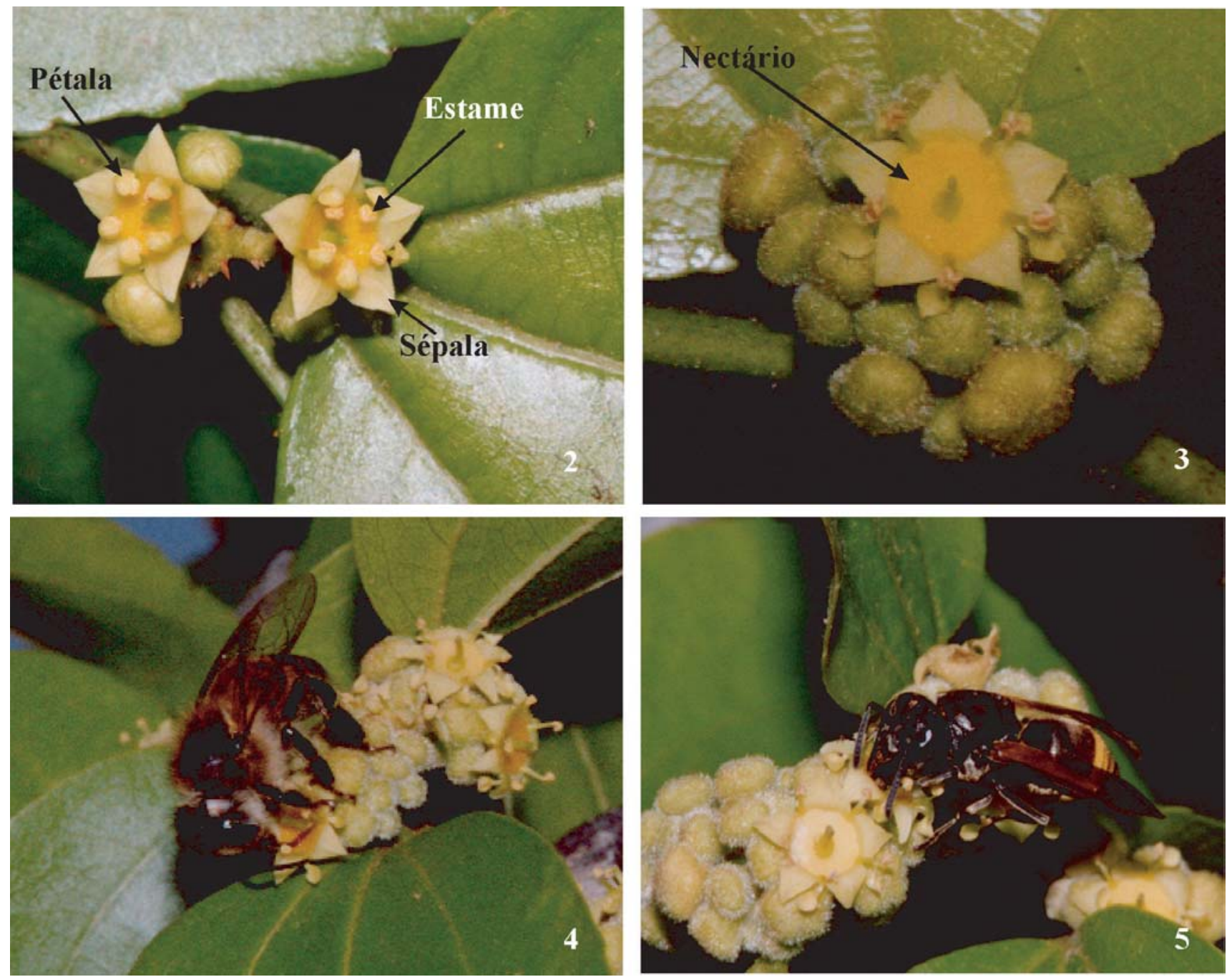

Figuras 2-5. Flores e visitantes florais de Ziziphus joazeiro Mart. (Rhamnaceae), na fazenda Dona Soledade, municípios de Boa Vista e Cabaceiras, Paraíba. Flores em fase masculina (2) e feminina (3). Visitas de Apis mellifera (4) e Brachygastra lecheguana (5). 
flores na fase feminina pela tarde. Por sua vez, indivíduos com flores que se abrem às $12: 00 \mathrm{~h}$ apresentam flores na fase masculina pela tarde e flores na fase feminina na manhã do dia seguinte. Conseqüentemente, enquanto um indivíduo inteiro apresenta flores na fase masculina, há, no mesmo horário, outro indivíduo com todas as flores na fase feminina. A fase feminina começa, então, em torno do meio dia em flores que se abrem pela manhã $(6: 00 \mathrm{~h})$ e por volta das 6:00 h do dia seguinte ao início de antese em flores que se abrem ao meio dia, estando o estigma receptivo nesses respectivos horários. Em nenhum momento foi observada sobreposição de fases masculina e feminina em um mesmo indivíduo. Na fase masculina, pétalas e estames estão perpendiculares ao receptáculo floral e o gineceu encontra-se bastante curto (Fig. 2). Nesse momento, as pétalas protegem os estames, cobrindo-os, sem, no entanto, impedir a exposição dos grãos de pólen. Ainda durante a fase masculina, as pétalas se movimentam até atingirem o mesmo nível das sépalas e o estilete se alonga. $\mathrm{Na}$ fase feminina (Fig. 3), as pétalas reflexas encontram-se no mesmo nível das sépalas, os estames começam a apresentar o mesmo movimento que as pétalas e os lobos do estigma tornam-se reflexos. As dimensões das estruturas florais variam de acordo com a fase sexual da flor (Tab. 1). A proporção de indivíduos com flores nas fases masculina e feminina na mesma população foi de 1:1 $(\mathrm{n}=10)$.

Após o período de antese, as flores permanecem na inflorescência por mais três ou quatro dias, apresentando nectário amarelo esbranquiçado não funcional, bem como anteras vazias e estigma não receptivo. Portanto, a duração de cada fase sexual em uma flor foi cerca de seis horas. As flores apresentam suave odor adocicado. Na fase masculina,

Tabela 1. Quantidade e dimensões das estruturas florais de Ziziphus joazeiro Mart. (Rhamnaceae), de acordo com as fases sexuais, masculina $\left(\sigma^{*}\right)$ e feminina ( $(+)$. Medidas feitas em uma população na fazenda Dona Soledade, municípios de Boa Vista e Cabaceiras, Paraíba. *Diâmetro.

\begin{tabular}{lccc}
\hline Caracteres florais & \multirow{2}{*}{ Quantidade } & \multicolumn{2}{c}{ Dimensões (mm) } \\
\cline { 3 - 4 } & & Fase ơc & Fase + \\
\hline Cálice (sépalas)* & 5 & 5 & 5 \\
Corola (pétalas) & 5 & 1 & 2 \\
Estames & 5 & 1 & 2 \\
Grãos de pólen/flor & $9.315( \pm 1.862)$ & - & - \\
Gineceu (carpelos/lóculos) & $2 / 2$ & - & 1 \\
Óvulos/flor & 2 & - & - \\
\hline
\end{tabular}

as margens das anteras e o nectário apresentaram reação positiva com o vermelho neutro. $\mathrm{Na}$ fase feminina, além das anteras e do nectário, todo o gineceu corou com solução de vermelho neutro.

A taxa de produção de néctar variou entre as flores que se abrem em diferentes horários. As flores que se abrem pela manhã produziram néctar durante as fases masculina e feminina, em volumes de, em média, $0,27 \pm 0,18 \mu \mathrm{l},(\mathrm{n}=10)$ e $0,05 \pm 0,06 \mu \mathrm{l},(\mathrm{n}=10)$, respectivamente, havendo diferença significativa entre as duas fases $(\mathrm{u}=7 ; \mathrm{p}=0,0012)$. As flores que se abrem pela tarde produziram néctar apenas na fase masculina, em média $0,15 \pm 0,07 \mu \mathrm{l}(\mathrm{n}=10)$, apresentando diferença em relação à fase masculina das flores que se abrem pela manhã $(u=24 ; p=0,0494)$. $\mathrm{O}$ volume de néctar acumulado durante todo o período de antese foi de $1 \mu \mathrm{l}$ para flores com antese de ambos horários, apresentando concentração de $75 \%$. O número de grãos de pólen por flor foi em média de $9.315 \pm 1.862(\mathrm{n}=5)$ e a viabilidade polínica média de $91 \%$.

Visitantes florais e disponibilidade polínica - Foram registradas oito espécies de vespas, cinco de moscas e seis de abelhas visitando flores de Ziziphus joazeiro (Tab. 2). Néctar foi o único recurso floral procurado pela maioria dos visitantes florais, exceto para Frieseomelitta flavicornis Fabricius que também coletava pólen. Ao coletar néctar, Apis mellifera Linné não tocava nas anteras ou estigma da flor (Fig. 4). No entanto, ao se deslocar pela inflorescência em direção à outra flor, esta abelha podia efetuar a transferência de pólen para o estigma, ficando os grãos de pólen aderidos em qualquer parte do corpo da abelha. Ao visitar pelo menos cinco vezes cada flor do experimento de exposição seletiva, Apis mellifera realizou a polinização em $78 \%$ das flores, sendo que $70 \%$ das flores visitadas apresentaram grãos de pólen emitindo tubo polínico.

As espécies de vespas contatavam anteras e estigma durante a coleta de néctar (Fig. 5), sendo o local de deposição de pólen mais definido, ficando os grãos de pólen aderidos às partes bucais do polinizador (Fig. 5). A espécie de vespa da subfamília Thynninae (Tiphiidae), no entanto, foi classificada como pilhadora de néctar, uma vez que raramente visitou as flores e apenas na fase masculina. As outras espécies de abelhas e todas as espécies de moscas pousavam nas flores para coleta de néctar, mas não contatavam as estruturas reprodutivas devido ao seu pequeno tamanho, sendo, então, pilhadoras. 
Tabela 2. Visitantes florais de Ziziphus joazeiro Mart. Comprimento do corpo, local de deposição do pólen (LDP), estádios de floração (EF) em que foram registrados, recursos coletados (RC) e resultado de suas visitas, na fazenda Dona Soledade, municípios de Boa Vista e Cabaceiras, Paraíba. $\mathrm{ND}=$ não definido, $\mathrm{PB}=$ partes bucais, $\mathrm{I}=$ início de floração, $\mathrm{P}$ = pico de floração, $\mathrm{F}=$ fim de floração, $\mathrm{N}=$ néctar, $\mathrm{PL}=$ pólen, $\mathrm{PO}=$ polinizador $\mathrm{PI}=$ pilhador.

\begin{tabular}{|c|c|c|c|c|c|}
\hline Visitantes & $\begin{array}{l}\text { Comprimento } \\
(\mathrm{mm})\end{array}$ & LDP & $\mathrm{EF}$ & $\mathrm{RC}$ & Classificação \\
\hline \multicolumn{6}{|l|}{ Abelhas } \\
\hline \multicolumn{6}{|l|}{ Apidae, Apinae, Apini } \\
\hline Apis mellifera Linné & 10 & ND & $\mathrm{I} / \mathrm{P} / \mathrm{F}$ & $\mathrm{N}$ & $\mathrm{PO}$ \\
\hline \multicolumn{6}{|l|}{ Apidae, Apinae, Meliponini } \\
\hline Frieseomelitta flavicornis Fabricius & 5 & - & $\mathrm{I} / \mathrm{P} / \mathrm{F}$ & $\mathrm{N}$ & PI \\
\hline Scaptotrigona sp. $\mathrm{n}$. & 5 & - & $\mathrm{P} / \mathrm{F}$ & $\mathrm{N}$ & PI \\
\hline Trigona fuscipennis (Friese) & 6 & - & $\mathrm{P} / \mathrm{F}$ & $\mathrm{N}$ & PI \\
\hline Trigonisca pediculana (Fabricius) & 2 & - & $\mathrm{I} / \mathrm{P} / \mathrm{F}$ & N/PL & PI \\
\hline \multicolumn{6}{|l|}{ Halictidae } \\
\hline $\mathrm{sp} .1$ & - & - & $\mathrm{F}$ & $\mathrm{N}$ & PI \\
\hline \multicolumn{6}{|l|}{ Vespas } \\
\hline \multicolumn{6}{|l|}{ Crabronidae } \\
\hline Larra sp. & 19 & $\mathrm{~PB}$ & $\mathrm{I} / \mathrm{P}$ & $\mathrm{N}$ & $\mathrm{PO}$ \\
\hline \multicolumn{6}{|l|}{ Tiphiidae, Thynninae } \\
\hline sp. 1 & 10 & - & I & $\mathrm{N}$ & PI \\
\hline \multicolumn{6}{|l|}{ Vespidae, Polistinae } \\
\hline Brachygastra lecheguana (Latreille) & 9 & $\mathrm{~PB}$ & $\mathrm{I} / \mathrm{P}$ & $\mathrm{N}$ & $\mathrm{PO}$ \\
\hline Polistes canadensis (L.) & 21 & $\mathrm{~PB}$ & $\mathrm{I} / \mathrm{P}$ & $\mathrm{N}$ & PO \\
\hline Polybia ignobilis (Haliday) & 13 & $\mathrm{~PB}$ & $\mathrm{I} / \mathrm{P}$ & $\mathrm{N}$ & PO \\
\hline Polybia ruficeps xanthops Richards & 9 & $\mathrm{~PB}$ & $\mathrm{I} / \mathrm{P}$ & $\mathrm{N}$ & $\mathrm{PO}$ \\
\hline Polybia sp. grupo occidentalis & 10 & $\mathrm{~PB}$ & $\mathrm{I} / \mathrm{P}$ & $\mathrm{N}$ & $\mathrm{PO}$ \\
\hline Protonectarina sylveirae (de Saussure) & 8 & $\mathrm{~PB}$ & $\mathrm{I} / \mathrm{P}$ & $\mathrm{N}$ & PO \\
\hline Moscas (5 spp.) & - & - & $\mathrm{I} / \mathrm{P} / \mathrm{F}$ & $\mathrm{N}$ & PI \\
\hline
\end{tabular}

O número de espécies visitantes e a freqüência das mesmas variaram de acordo com o estádio de floração de Ziziphus joazeiro. No início e pico de floração ocorreu o maior número de espécies visitantes (12 e 13 espécies, respectivamente, considerando as diferentes espécies de moscas como um único grupo). No final da floração foram observadas apenas sete espécies visitantes, incluindo o grupo de moscas. Apis mellifera contribuiu com o maior número de visitas, tanto no início como no pico de floração, correspondendo a $56,2 \%$ e $77,5 \%$ do total de visitas, respectivamente (Fig. 6). Polybia ruficeps xanthops Richards (Vespidae, Polistinae) foi a segunda espécie que mais contribuiu com número de visitas no início da floração $(10,6 \%)$, reduzindo consideravelmente sua frequiência no pico de floração $(0,8 \%)$. Brachygastra lecheguana (Latreille) e Polybia ignobilis (Haliday) (Vespidae, Polistinae) mantiveram aproximadamente o mesmo percentual de visitas (ca. 8\% e 6\%, respectivamente) no início e pico de floração (Fig. 6). Esses últimos foram, portanto, os visitantes que mais contribuíram em termos de percentual de visitas durante a floração de Ziziphus joazeiro, excetuando as visitas por Apis mellifera. Os outros visitantes florais foram pouco freqüentes, cada um contribuindo com menos de $5 \%$ do total de visitas. No final da

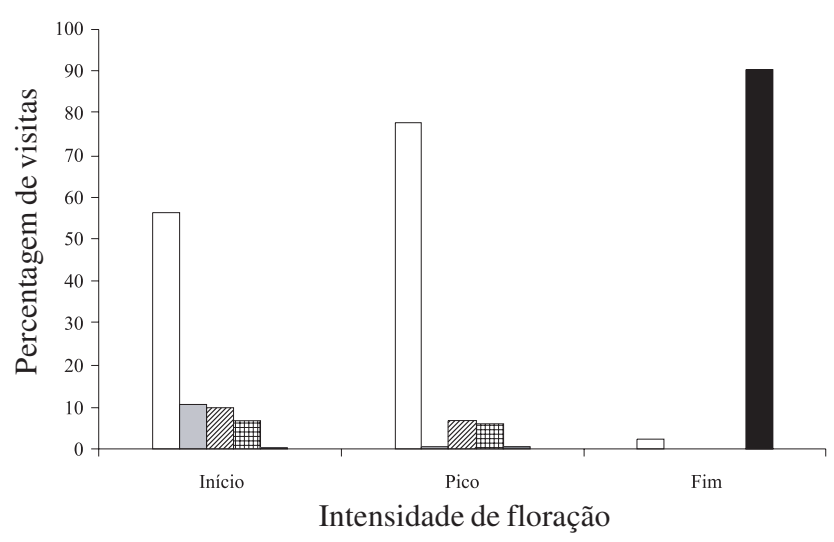

Figura 6. Percentual de visitas às flores de Ziziphus joazeiro Mart. (Rhamnaceae) dos principais visitantes no início, pico e fim de floração, na fazenda Dona Soledade, municípios de Boa Vista e Cabaceiras, Paraíba. Apis mellifera Linné ( $\square$ ), Polybia ruficeps xanthops Richards ( $\square$ ), Brachygastra lecheguana (Latreille) (ש/d), Polybia ignobilis (Haliday) (N), Frieseomelitta flavicornis Fabricius (ם). 
floração, Frieseomelitta flavicornis foi responsável por $90,3 \%$ do total de visitas (Fig. 6). No entanto, de acordo com o seu comportamento, não realiza a polinização.

As visitas começavam após as 6:00 h, não mais ocorrendo após as 17:45 h (Fig. 7). O período de maior freqüência de visitas ocorreu entre 11:00 h e 14:00 h no início, 9:00 h e 11:00 h no pico, e 9:00 h e 12:00 h no fim de floração (Fig. 7). Apis mellifera, Brachygastra lecheguana e Poybia ignobilis visitaram as flores de Ziziphus joazeiro ao longo do dia, tanto no início como no pico da floração. No final da floração, apenas Apis mellifera, entre os polinizadores, foi observada forrageando no período de 8:00 h às 9:00 h. Os outros polinizadores apresentaram horário mais restrito de visitas. Nos três estádios de floração, houve registro de pelo menos uma espécie pilhadora visitando flores de Z. joazeiro ao longo de todo o dia, havendo maior predomínio de pilhadores no final da floração. Todas as espécies de animais foram observadas visitando apenas flores funcionais (dentro das 12 horas de antese), tanto na fase masculina quanto na fase feminina, havendo maior número de visitas em flores na fase feminina $\left(\chi^{2}=289, \mathrm{gl}=1, \mathrm{p}<0,001\right)$. Esse fato pode ser devido à menor quantidade de néctar produzida nessa fase, necessitando que haja um maior número de visitas para o suprimento alimentar dos visitantes.

O número de grãos de pólen por flor diminuiu acentuadamente nas três primeiras horas de antese em flores que se abrem ao meio dia (Fig. 8). No período da tarde, a frequiência de visitas é maior logo nos primeiros horários (ver Fig. 7), ocasionando essa

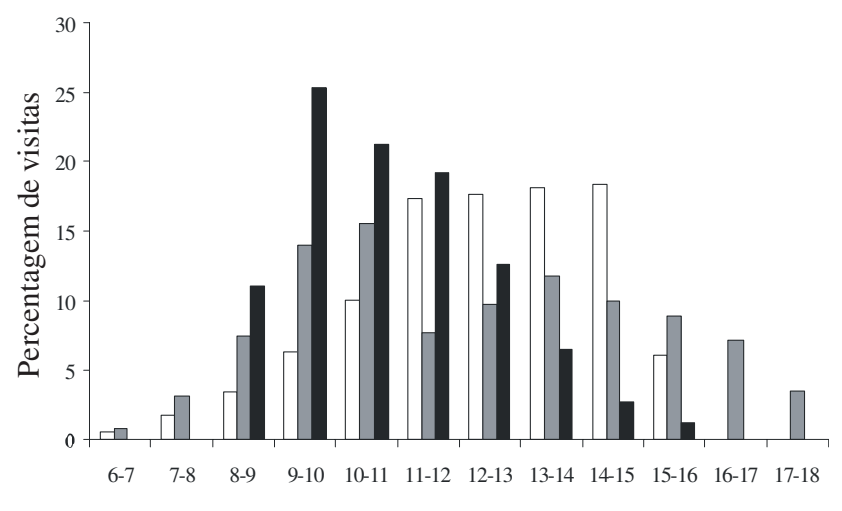

Hora

Figura 7. Percentual de visitas, de todos os visitantes florais, às flores de Ziziphus joazeiro Mart. (Rhamnaceae) ao longo do dia em indivíduos no início ( $\square$ ), pico ( $\square$ ) e fim ( $\square$ ) de floração, na fazenda Dona Soledade, municípios de Boa Vista e Cabaceiras, Paraíba.

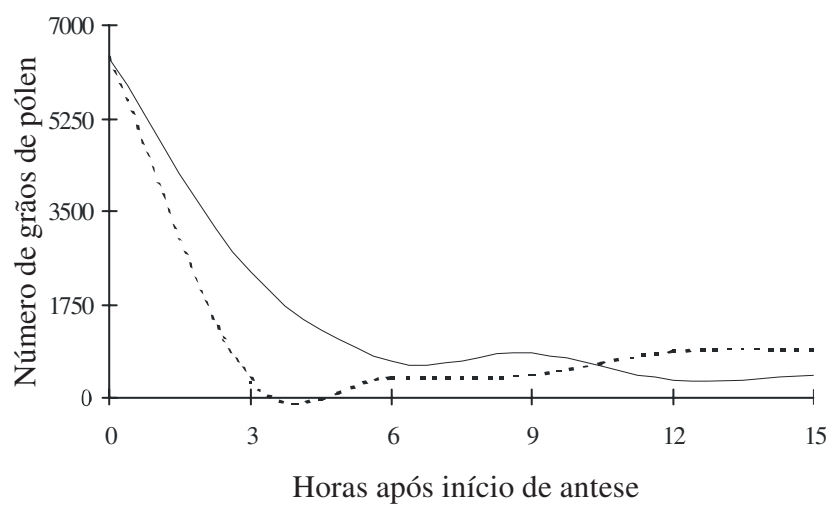

Figura 8. Disponibilidade de grãos de pólen durante a antese em flores ( $\mathrm{n}=10$ /indivíduo) de Ziziphus joazeiro Mart. (Rhamnaceae) que abrem no período da manhã (-) ou ao meio dia (---), na fazenda Dona Soledade, municípios de Boa Vista e Cabaceiras, Paraíba.

acentuada queda na disponibilidade de grãos de pólen. Em flores que se abrem pela manhã, ocorreu maior redução na quantidade de grãos de pólen dentro das seis primeiras horas de antese, correspondendo à duração da fase masculina (Fig. 8), talvez em decorrência da maior frequiência de visitas ocorrer apenas no fim da manhã (ver Fig. 7). O fato de, na fase feminina, as anteras ainda apresentarem alguma quantidade de grãos de pólen pode permitir que ocorra a geitonogamia mediada pelos polinizadores ao se deslocarem pela inflorescência.

Eficácia reprodutiva - $\mathrm{O}$ sucesso reprodutivo na polinização cruzada não apresentou diferença em relação à polinização natural, apresentando índice de eficácia reprodutiva de 0,4 (Tab. 3). O número de frutos formados por inflorescência variou entre um e dois, havendo, raramente, inflorescências com três frutos, não havendo correlação entre o número de flores e de frutos por inflorescência.

Tabela 3. Total de frutos formados por inflorescência e sucesso reprodutivo de Ziziphus joazeiro Mart. (Rhamnaceae) sob condições naturais e através de polinização cruzada manual para análise da eficácia reprodutiva, na fazenda Dona Soledade, municípios de Boa Vista e Cabaceiras, Paraíba. ${ }^{a}$ Diferença não significativa.

Tratamento Inflorescências Flores/Frutos Sucesso

$\begin{array}{lccc}\text { Polinização natural } & 30 & 781 / 10 & 1,3^{\mathrm{a}} \\ \text { Polinização cruzada } & 15 & 67 / 2 & 3^{\mathrm{a}} \\ \text { Eficácia reprodutiva } & & & 0,4\end{array}$




\section{Discussão}

Fenologia reprodutiva e biologia floral - Ziziphus joazeiro apresentou padrão de floração do tipo cornucópia de acordo com a classificação de Gentry (1974), uma vez que produziu muitas flores ao longo de várias semanas, apresentando sazonalidade. A floração de $Z$. joazeiro também pode ser classificada como anual, segundo classificação de Newstrom et al. (1994), pois apresentou apenas um ciclo no período de um ano. Alguns trabalhos registraram sazonalidade na floração e frutificação em espécies da Caatinga, sendo estas influenciadas principalmente pela precipitação e ocorrendo, na maioria delas, na estação chuvosa (Machado et al. 1997; Griz \& Machado 2001; Barbosa et al. 2003). A floração e frutificação ocorreram no fim do período seco e início do período chuvoso, respectivamente, estando de acordo com o encontrado por Barbosa et al. (1989), Pinheiro et al. (1991), Machado et al. (1997) e Griz \& Machado (2001). No entanto, Pinheiro et al. (1991) registraram que os indivíduos cujas flores se abriam pela manhã floresceram dois meses antes dos indivíduos em que as flores se abriam à tarde. Essa diferença não ocorreu na área do presente estudo. A ocorrência de frutificação no período chuvoso pode favorecer a dispersão das sementes e o estabelecimento de plântulas (Bawa 1983; Primack 1987). De fato, os frutos de Z. joazeiro são zoocóricos (Griz \& Machado 2001) e dependem de animais para dispersão a longa distância, podendo o período de chuvas ser importante para a germinação e o recrutamento.

$\mathrm{O}$ fato de as flores de $Z$. joazeiro permanecerem (mesmo que com anteras vazias e com nectário e estigma não funcionais) na inflorescência após o período de antese pode aumentar a atração floral para os polinizadores, uma vez que o padrão de floração pode influenciar a longevidade floral (Primack 1985). Espécies que apresentam padrão cornucópia (sensu Gentry 1974) geralmente possuem flores que duram apenas um dia, produzem néctar como recurso floral e são visitadas por amplo espectro de visitantes florais (Primack 1985). Ziziphus joazeiro apresentou todas essas características.

A seqüência de antese e as fases florais de Ziziphus joazeiro descritas neste trabalho já haviam sido mencionadas de forma semelhante por Pinheiro et al. (1991). Ziziphus spina-christi (L.) Willd. (Galil \& Zeroni 1967) e Z. mucronata Willd. (Zietsman 1990) também apresentaram protandria com fases florais semelhantes a $Z$. joazeiro, com dois grupos de indivíduos com horários diferentes de abertura das flores. Essa diferença no horário de antese favorece a polinização cruzada, uma vez que em um mesmo indivíduo não há sobreposição de fases sexuais, minimizando as chances de que ocorra geitonogamia (Lloyd \& Webb 1986). Pinheiro et al. (1991) ainda relatam sobreposição das fases sexuais em $Z$. joazeiro no final de floração, o que, no entanto, não foi observado no presente estudo. A presença desses dois grupos de indivíduos, juntamente com a ocorrência de dicogamia, caracteriza um tipo de heterodicogamia, descrito por Lloyd \& Webb (1986) e que tem sido relatada para um total de 11 famílias e 17 gêneros (Renner 2001). Além das espécies de Ziziphus citadas anteriormente, $Z$. jujuba e Z. mauritiana também apresentam heterodicogamia (Renner 2001), podendo ser uma característica comum para o gênero.

A produção de néctar foi maior na fase masculina, diferente do encontrado por Pinheiro et al. (1991). Essas autoras observaram "baixa" produção de néctar no início da antese (correspondendo à fase masculina), atingindo o máximo na fase feminina. No entanto, não houve quantificação do volume de néctar no referido estudo. Na população estudada, ao contrário do relatado por Pinheiro et al. (1991), flores na fase feminina produziram menor quantidade de néctar ou, dependendo do tipo de indivíduo, não produziram néctar. A produção de pequena quantidade de néctar é característica de flores polinizadas por pequenos animais (Faegri \& Pijl 1979), como é o caso de Z. joazeiro. Além disso, essa característica induz à procura por um maior número de flores e, conseqüentemente, associada à heterodicogamia, aumenta as chances de polinização cruzada (Cruden et al. 1983).

A concentração de açúcares no néctar também está relacionada com o tipo de visitante floral (Baker 1975; Faegri \& Pijl 1979; Proctor et al. 1996). Segundo Baker (1975), flores visitadas por abelhas e vespas apresentam alta concentração de açúcares no néctar (13-50\%), o que foi encontrado para $Z$. joazeiro no presente estudo, tendo concentração maior $(75 \%)$ do que o intervalo estabelecido por Baker (1975). Além do volume e da concentração de açúcares no néctar, $Z$. joazeiro também apresentou outras características comuns às espécies polinizadas por vespas, tais como flores claras, com corola curta, que permite fácil acesso ao néctar, e emissão de odor adocicado (sensu Faegri \& Pijl 1979; Endress 1994; Proctor et al. 1996). Tais atributos, incluindo o valor da concentração de açúcares no néctar, foram também relatados por Santos 
\& Machado (1998) para Vismia guianensis (Aubl.) Choisy (Gutiferae), polinizada por abelhas e vespa, as quais registraram concentração de 44 a $60 \%$.

Visitantes florais - Apesar de Apis mellifera contatar as estruturas reprodutivas da flor apenas durante seu deslocamento de uma flor para outra, essa abelha foi considerada como importante polinizador de $Z$. joazeiro devido à sua alta freqüência de visitas e ao alto percentual de deposição de pólen nos estigmas. No estudo de Pinheiro et al. (1991), essa abelha foi considerada como único polinizador efetivo de Z. joazeiro, as quais não registraram vespas. No presente estudo, as vespas, possuindo aparelho bucal mais curto que $A$. mellifera, efetuavam a polinização tocando nas anteras e no estigma durante a coleta de néctar em cada flor, sendo os polinizadores autóctones da espécie.

Entre as espécies de vespas, Brachygastra lecheguana e Polybia ignobilis foram consideradas os principais polinizadores de Z. joazeiro, pois estas, além do tamanho e comportamento adequados à polinização, contribuíram com maior percentual de visitas ao longo de todo o dia. Todos os polinizadores de Ziziphus joazeiro, excetuando-se Apis mellifera, são espécies nativas da região, sendo $Z$. joazeiro uma importante fonte de recurso alimentar.

No que diz respeito à relevância de Apis mellifera para a polinização de Ziziphus joazeiro, esta teve papel importante no local de estudo principalmente no final da floração, quando não foram registradas visitas dos polinizadores nativos. No entanto, a baixa freqüência ou ausência desses últimos durante a floração de Z. joazeiro pode já ser decorrência da alta freqüência de Apis mellifera. Redução na freqüência de visitas de polinizadores nativos em decorrência de interações agonísticas causadas por A. mellifera foi relatada em outros estudos (Paton 1993; Gross \& Mackay 1998). Portanto, é possível que esta abelha esteja afetando negativamente a disponibilidade de recurso alimentar para as vespas. Comparações, utilizando metodologias similares, em situações onde apenas A. mellifera ou vespas atuem como polinizadores e quando os dois grupos estão presentes serão relevantes para se verificar o efeito da presença desta abelha no sucesso reprodutivo de Z. joazeiro. Alguns estudos mostram déficit no sucesso reprodutivo de plantas quando A. mellifera ocorre em conjunto com polinizadores nativos (Gross \& Mackay 1998; Carmo et al. 2004; Paton 1993).

Abelhas da tribo Meliponini foram encontradas na população em estudo visitando as flores de Ziziphus joazeiro, fato também registrado por Marinho et al. (2002) os quais reportaram esta espécie como importante recurso alimentar para espécies desta tribo. No entanto, no presente trabalho, as Meliponini não foram freqüentes e não realizavam a polinização em Z. joazeiro.

Eficácia reprodutiva - $\mathrm{O}$ número de frutos formados naturalmente foi bem menor do que o número de flores produzidas e, segundo Stephenson (1981), a produção excessiva de flores, como no caso de Ziziphus joazeiro, pode estar relacionada a algumas estratégias que aumentam o "fitness" da planta, tais como aumento da atratividade para o polinizador ou aborto seletivo de frutos, considerados como "de baixa qualidade". Dificilmente o baixo percentual de frutos formados sob condições naturais em $Z$. joazeiro seria resultado da baixa eficiência de polinizadores, apesar dessa espécie ter apresentado eficácia reprodutiva relativamente baixa, que refletiria a eficiência dos polinizadores (Zapata \& Arroyo 1978). Um indicativo para a alta eficiência dos polinizadores em $Z$. joazeiro pode ser o fato da espécie ter apresentado, algumas vezes, até cinco ou mais frutos em estágio de desenvolvimento inicial em uma única inflorescência. Como na fase de maturação de frutos cada inflorescência produz de um a dois frutos, raramente três, a baixa produção de frutos realmente parece estar relacionada com o aborto de frutos jovens.

Desse modo, os polinizadores, autóctones ou não, de Ziziphus joazeiro têm papel fundamental para a sua reprodução sexuada, uma vez que a baixa quantidade de néctar por flor induz a um aumento no número de visitas, minimiza as chances de geitonogamia e a heterodicogamia presente na espécie impossibilita a formação de frutos por autopolinização espontânea.

\section{Agradecimentos}

Aos Drs. Ana Virgínia Leite (UFPE) e André Santos (UFPE), pela constante ajuda nas atividades de campo; à Dona Ana Maria Almeida, pela permissão em desenvolver este trabalho em áreas de sua propriedade; ao Dr. Orlando Tobias Silveira (MPEG/PA), pela gentileza na identificação das vespas; às Dras. Patrícia Albuquerque e Márcia Rego (UFMA), pela identificação das abelhas; ao Governo da Paraíba, por disponibilizar os dados pluviométricos na internet; à CAPES, pela bolsa de mestrado concedida à primeira autora; ao $\mathrm{CNPq}$, pelo apoio financeiro através do Edital Universal 2004 (processo 
n. 475778/2004-9) e das bolsas de Produtividade em Pesquisa de I. Machado e A. Lopes.

\section{Referências bibliográficas}

Aguiar, C.M.L.; Zanella, F.C.V.; Martins, C.F. \& Carvalho, C.A.L. 2003. Plantas visitadas por Centris spp. (Hymenoptera: Apidae) na caatinga para obtenção de recursos florais. Neotropical Entomology 32: 247-259.

Ayres, M.; Ayres Jr., M.; Ayres, D.L. \& Santos, A.S. 2003. BioEstat 3.0. Aplicações estatísticas nas áreas das ciências biológicas e médicas. Sociedade Civil Mamirauá /MCT-CNPq /Conservation International.

Baker, H.G. 1975. Sugar concentration in nectars from hummingbird flowers. Biotropica 7: 37-41.

Barbosa, D.C.A.; Alves, J.L.H.; Prazeres, S.M. \& Paiva, A.M.A. 1989. Dados fenológicos de 10 espécies arbóreas de uma área de caatinga (Alagoinha-PE). Acta Botanica Brasilica 3: 109-117.

Barbosa, D.C.A.; Barbosa, M.C.A. \& Lima, L.C.M. 2003. Fenologia de espécies lenhosas da caatinga. Pp. $657-$ 693. In: I.R. Leal; M. Tabarelli \& J.M.C. Silva (eds.). Ecologia e Conservação da Caatinga. Recife, Universidade Federal de Pernambuco.

Barros, N.N.; Freire, L.C.L.; Lopes, E.A. \& Johnson, W.L. 1991. Valor nutritivo do feno de juazeiro (Zizyphus joazeiro) para caprinos e ovinos. Pesquisa Agropecuária Brasileira 26: 1299-1304.

Bawa, K.S. 1983. Patterns of flowering in tropical plants. Pp. 394-410. In: C.E. Jones \& R.J. Little (eds.). Handbook of Experimental Pollination Biology. New York, Van Nostrand Reinhold.

Bencke, C.S.C. \& Morellato, L.P.C. 2002. Comparação de dois métodos de avaliação da fenologia de plantas, sua interpretação e representação. Revista Brasileira de Botânica 25: 269-275.

Braga, R. 1976. Plantas do Nordeste, especialmente do Ceará. Natal, ESAM.

Carmo, R.M.; Franceschinelli, E.V. \& Silveira, F.A. 2004. Introduced honeybees (Apis mellifera) reduce pollination success without affecting the floral resource taken by native pollinators. Biotropica 36: 371-376.

CNIP - Centro Nordestinho de Informações sobre Plantas. [on line] Disponível na Internet via www.url: http:// www.cnip.org.br. (Acesso: 02/julho/2005).

Cruden, R.W. 1977. Pollen-ovule ratios: A conservative indicator of breeding systems in flowering plants. Evolution 31: 32-46.

Cruden, R.W.; Hermann, S.M. \& Peterson, S. 1983. Patterns of nectar production and plant-pollinator coevolution. Pp. 80-125. In: B. Bentley \& T. Elias (eds.). The Biology of Nectaries. New York, Columbia University Press.

Dafni, A. 1992. Pollination ecology: a practical approach. New York, Oxford University Press.

Endress, P.K. 1994. Diversity and evolutionary biology of tropical flowers. Cambridge, Cambridge University Press.
Faegri, K. \& Pijl, L.van der. 1979. The principles of pollination ecology. Oxford, Pergamon Press.

Fournier, L.A. 1974. Un metodo cuantitativo para la medición de características fenologicas en árboles. Turrialba 24: 422-423.

Galil, J. \& Zeroni, M. 1967. On the pollination of Zizyphus spina-christi (L.) Willd. in Israel. Israel Journal of Botany 16: 71-77.

Gentry, A.H. 1974. Flowering phenology and diversity in tropical Bignoniaceae. Biotropica 6: 64-68.

Giulietti, A.M.; Harley, R.M.; Queiroz, L.P.; Barbosa, M.R.V.; Bocage Neta, A.L. \& Figueiredo, M.A. 2002. Espécies endêmicas da caatinga. Pp. 103-115. In: E.V.S.B. Sampaio; A.M. Giulietti; J. Virgínio \& C.F.L. Gamarra-Rojas (eds.). Vegetação \& Flora da Caatinga. Recife, Associação Plantas do Nordeste - APNE, Centro Nordestino de Informação sobre Plantas - CNIP.

Governo da Paraíba. [on line]. Disponível na Internet via www.url: http://www.paraiba.pb.gov.br. (Acesso: 15/março/2005).

Griz, L.M.S. \& Machado, I.C.S. 2001. Fruiting phenology and seed dispersal syndromes in caatinga, a tropical dry forest in the northeast of Brazil. Journal of Tropical Ecology 17: 303-321.

Gross, C.L. \& Mackay, D. 1998. Honeybees reduce fitness in the pioneer shrub Melastoma affine (Melastomataceae). Biological Conservation 86: 169-178.

Heald, S.V. 2004. Rhamnaceae. Pp. 323-324. In: N. Smith; S.A. Mori; A. Henderson; D.W. Stevenson \& S.V. Heald (eds.). Flowering Plants of the Neotropics. New Jersey, Princeton University Press.

Kearns, C.A. \& Inouye, D.W. 1993. Techniques for pollination biologists. Colorado, University Press of Colorado.

Lloyd, D.G. \& Webb, C.J. 1986. The avoidance of interference between the presentation of pollen and stigmas in angiosperms. New Zealand Journal of Botany 24: 135-162.

Lorenzi, H. 1998. Zizyphus joazeiro. Pp. 299. In: H. Lorenzi (ed.). Árvores Brasileiras: Manual de Identificação e Cultivo de Plantas Arbóreas do Brasil. v.1. Nova Odessa, Editora Plantarum.

Machado, I.C.S.; Barros, L.M. \& Sampaio, E.V.S.B. 1997. Phenology of caatinga species at Serra Talhada, PE, northeastern Brasil. Biotropica 29: 57-68.

Marinho, I.V.; Freitas, M.F.; Zanella, F.C.V. \& Caldas, A.L. 2002. Espécies vegetais da caatinga utilizadas pelas abelhas indígenas sem ferrão como fonte de recursos e local de nidificação. In: Anais I Congresso Brasileiro de Extensão Universitária. João Pessoa.

Martin, F.W. 1959. Staining and observing pollen tubes in the style by means of fluorescence. Stain Technology 34: $125-128$.

Newstrom, L.E.; Frankie, G.W. \& Baker, H.G. 1994. A new classification for plant phenology based on flowering patterns in lowland Tropical Rain Forest Trees at La Selva, Costa Rica. Biotropica 26: 141-159.

Paton, D.C. 1993. Honeybees in the Australian environment. BioScience 43: 95-103. 
Pinheiro, M.C.B.; Ormond, W.T. \& Castro, A.C. 1991. Biologia da reprodução e fenologia de Ziziphus joazeiro Mart. (Rhamnaceae). Revista Brasileira de Biologia 51: 143-152.

Prado, D.E. 2003. As caatingas da América do Sul. Pp. 3-73. In: I.R. Leal; M. Tabarelli \& J.M.C. Silva (eds.). Ecologia e Conservação da Caatinga. Recife, Universidade Federal de Pernambuco.

Primack, R.B. 1985. Longevity of individual flowers. Annual Review of Ecology and Systematics 16: 15-37.

Primack, R.B. 1987. Relationships among flowers, fruits, and seeds. Annual Review of Ecology and Systematics 18: 409-430.

Proctor, M.; Yeo, P. \& Lack, A. 1996. The natural history of pollination. Oregon, Timber Press.

Radford, A.E.; Dickinson, W.C.; Massey, J.R. \& Bell, C.R. 1974. Vascular plant systematics. New York, Harper \& Row Publishers.

Renner, S.S. 2001. How common is heterodichogamy? Trends in Ecology \& Evolution 16: 595-597.
Robinsohn, I. 1924. Die farbungsreaktion der narbe, stigmatochromie, als morpho-biologische Blütenuntersuchungsmethode. Sitzungsberichten Akademischer Wissenschaft Wien, Mathematics, Abteilung 133: 180-213.

Santos, M.J.L. \& Machado, I.C. 1998. Biologia floral e heterostilia em Vismia guianensis (Aubl.) Choisy (Clusiaceae). Acta Botanica Brasilica 12: 451-464.

Stephenson, A.G. 1981. Flower and fruit abortion: proximate causes and ultimate functions. Annual Review of Ecology and Systematics 12: 253-279.

Vogel, S. 1990. The role of scent glands in pollination. New Delhi, Amerind Publishing.

Zapata, T.R. \& Arroyo, M.T.K. 1978. Plant reproductive ecology of a secondary deciduous tropical forest in Venezuela. Biotropica 10: 221-230.

Ziestman, P.C. 1990. Pollination of Ziziphus mucronata subsp. mucronata (Rhamnaceae). Suid Afrikaanse Tydskrift Plantk 56: 350-355. 